\title{
Sunitinib Inhibits Inflammatory Corneal Lymphangiogenesis
}

\author{
Benoît Detry, ${ }^{1}$ Silvia Blacher, ${ }^{1}$ Charlotte Erpicum, ${ }^{1}$ Jenny Paupert,${ }^{1}$ Ludovic Maertens, ${ }^{1}$ \\ Catherine Maillard, ${ }^{1}$ Carine Munaut, ${ }^{1}$ Nor Eddine Sounni, ${ }^{1}$ Vincent Lambert,,${ }^{1,2}$ \\ Jean-Michel Foidart, ${ }^{1}$ Jean-Marie Rakic, ${ }^{2}$ Didier Cataldo, ${ }^{1}$ and Agnès Noël ${ }^{1}$
}

${ }^{1}$ Laboratory of Tumor and Developmental Biology, Groupe Interdisciplinaire de Génoprotéomique Appliqué-Recherche (GIGACancer), University of Liège, Liège, Belgium

${ }^{2}$ Department of Ophthalmology, Centre Hospitalier Universitaire, Liège, Belgium

Correspondence: Agnès Noël, Laboratory of Tumor and Developmental Biology, University of Liège, Tour de Pathologie, CHU (B23), Sart Tilman, B-4000 Liège, Belgium; agnes.noel@ulg.ac.be.

Submitted: August 27, 2012 Accepted: April 4, 2013

Citation: Detry B, Blacher S, Erpicum C, et al. Sunitinib inhibits inflammatory corneal lymphangiogenesis. Invest Ophthalmol Vis Sci. 2013;54:3082-3093. DOI:10.1167/ iovs.12-10856
Purpose. To evaluate the antilymphangiogenic potential of multi-target tyrosine kinase inhibitor sunitinib in corneal neovascularization (NV).

Methods. Inflammatory corneal NV was induced by thermal cauterization applied in the central cornea of mice, to which sunitinib malate was daily administered by gavage or not. At days 6,11 , or 17 post cauterization, lymphatic and blood vessels, as well as inflammatory cells were immunostained and quantified in whole-mounted corneas. RT-PCRs were performed to evidence VEGF-A, VEGF-C, VEGF-D, placental growth factor (PIGF), and soluble vascular endothelial growth factor receptor (VEGFR)-1 and -2 (sVEGFR-1, sVEGFR-2) expressions. Macrophages were isolated from mice peritoneal cavity following thioglycollate injection to produce conditioned medium. The effects of sunitinib were evaluated in vitro in the aortic and lymphatic ring assays in the presence or not of macrophage conditioned medium.

Results. Sunitinib treatment drastically reduced pathologic corneal lymphangiogenesis and angiogenesis. Reduced $\mathrm{F} 4 / 80+$ cell infiltration was evidenced in sunitinib-treated mice and was associated to decreased VEGF-A (by 50\%, $P<0.01$ ) and VEGF-C (by 35\%, $P<0.01$ ) expressions, while VEGF-D and sVEGFR-2 expressions were not affected. In vitro, sunitinib dose-dependently inhibited aortic ring outgrowth, but failed to affect lymphangiogenesis in the lymphatic ring assay. However, macrophage conditioned medium-enhanced angiogenesis and lymphangiogenesis were both strongly counteracted by sunitinib treatment. Mechanistically, sunitinib blocked VEGFR-2 phosphorylation induced by VEGF-A released by macrophages.

Conclusions. Sunitinib exerts antihemangiogenic and antilymphangiogenic effects in vivo by reducing $\mathrm{F} 4 / 80+$ cell recruitment and interacting with their released factors.

Keywords: corneal neovascularization, sunitinib, lymphangiogenesis
$T_{\mathrm{h}}^{\mathrm{h}}$ he avascularity of the cornea is actively maintained in healthy conditions and is necessary to preserve cornea transparency and optimal visual acuity. ${ }^{1}$ This "angiogenic privilege" relies on the production of antilymph/angiogenic factors such as soluble forms of vascular endothelial growth factor receptor (sVEGFR-1, sVEGFR-2), ${ }^{2-4}$ thrombospondins, ${ }^{5}$ angiostatin, endostatin, and pigmented epithelium derived factor, ${ }^{6}$ counterbalancing the basal expression of prolymph/ angiogenic factors (e.g., VEGF-A). ${ }^{1}$ The avascular cornea can secondarily be invaded by blood vessels (hemangiogenesis) and/or lymphatic vessels (lymphangiogenesis) under severe inflammation. Thus, such a corneal neovascularization (NV) can occur in several pathologic conditions such as hypoxia, infections (e.g., Herpes Simplex Virus, Chlamydia trachomatis), trauma, or keratoplasty, leading to reduced visual acuity. ${ }^{7}$ $\mathrm{NV}$, in particular lymphangiogenesis, is also the major factor leading to cornea graft rejection occurring after keratoplasty. ${ }^{8}$

The VEGF family members are generally considered as the main regulators of lymph/angiogenesis through the binding to their receptors, the VEGFRs. Among them, VEGF-A enhances hemangiogenesis through VEGFR-2 binding, expressed by blood endothelial cells. VEGF-C and -D are the main prolymphangiogenic factors that act through the activation of VEGFR-3 expressed on lymphatic endothelial cells (LECs). However, LECs also express VEGFR-2 and processed forms of VEGF-C and VEGF-D are also able to bind VEGFR-2, demonstrating interconnections between the two pathways. ${ }^{9}$ VEGF-A-mediated corneal $\mathrm{NV}$ has been attributed to the stimulation of both hemangiogenesis and lymphangiogenesis, ${ }^{7,10}$ directly ${ }^{11}$ or indirectly by recruiting inflammatory cells able to deliver VEGF-C and $-\mathrm{D} .{ }^{12}$

Strategies aiming at blocking VEGF pathway have been developed to inhibit lymph/angiogenesis, and notably consist of anti-VEGF-A antibodies and aptamers (bevacizumab, ranibizumab, pegaptanib) or small molecules designed to inhibit the tyrosine kinase activity of VEGFRs (sunitinib, sorafenib, vatalanib, etc.). Although bevacizumab was approved by Food and Drug Administration (FDA) for cancer and AMD treatment, ${ }^{13}$ and several molecules including anti-VEGF antibodies and tyrosine kinase inhibitors have been demonstrated to efficiently inhibit corneal $\mathrm{NV}$ in mice models, ${ }^{14-16}$ no treatment specifically targeting lymph/angiogenesis is available to treat 
corneal NV. ${ }^{7}$ Corneal NV is currently treated with corticosteroids, which control inflammatory reaction and display antilymph/angiogenic properties, but their effects are limited and are associated to adverse effects. ${ }^{7,17}$

In the present work, we evaluated the impact of sunitinib on corneal NV. Sunitinib is a multi-target tyrosine kinase inhibitor able to inhibit VEGFRs, platelet-derived growth factor receptor (PDGFR), c-kit and ret, and has already been approved by FDA for the treatment of metastasic renal cell carcinomas and gastro intestinal stromal tumors that are resistant to imatinib. ${ }^{18}$ Although antiangiogenic effects of sunitinib through VEGFR-2 inhibition is well recognized, and has been shown in rabbit corneas, ${ }^{19}$ its effect on lymphangiogenesis remains unclear. Kodera and colleagues recently demonstrated sunitinib-inhibited lymphangiogenesis in a murine model of breast cancer through VEGFR-3 inhibition. ${ }^{20}$ However, whether sunitinib is or is not a potent inhibitor of corneal lymphangiogenesis is still unknown. We demonstrate here that sunitinib exerts in vivo earlier and stronger inhibitory effects on corneal lymphangiogenesis than on hemangiogenesis. These inhibitory effects are associated with reduced $\mathrm{F} 4 / 80+$ cell recruitment. In vitro studies underscore an indirect effect on lymphangiogenesis through the interference with lymphangiogenic factors released by macrophages.

\section{Materials AND Methods}

\section{Mice}

Six-week-old male C57BL/6 mice purchased from Janvier (Saint Berthevin, France) were used throughout this study. The animals were maintained with a 12 hour light-dark cycle and had free access to food and water. Animal experiments were performed in compliance with the Animal Ethical Committee of the University of Liège (Liège, Belgium) and in accordance with the ARVO Statement for the Use of Animals in Ophthalmic and Vision Research.

\section{Sunitinib}

Sunitinib malate (SU-11248) was purchased from LC Laboratories (Woburn, MA). For in vivo assays, sunitinib was solubilized in $0.5 \%$ carboxymethylcellulose and $40 \mathrm{mg} / \mathrm{kg}$ were daily administered by oral gavage. Control mice received vehicle. For in vitro assays, sunitinib was solubilized in dimethyl sulfoxide (DMSO) and used at $20 \mathrm{nM}$ in culture medium containing $0.1 \%$ DMSO.

\section{Corneal Neovascularization Assay and Immunostainings}

Corneal NV was induced by thermal cauterization as previously described. ${ }^{21-23}$ After anesthetizing the mouse with intraperitoneal injection of ketamine hydrochloride $(100 \mathrm{mg} / \mathrm{kg}$ body weight) and xylazine (10 mg/kg body weight), the anesthetized central cornea (Unicaine $0.4 \%$; Thea Pharma, Wetteren, Belgium) was thermally cauterized using an ophthalmic cautery (Optemp II V; Alcon Surgical, Fort Worth, TX). Mice were time course sacrificed and eyes were removed and dissected. Whole-mounted corneas were fixed in $70 \%$ ethanol for 1 hour at room temperature and blocked in 3\% BSA-3\% Gloria milk (Nestlé, Brussels, Belgium) for 1 hour.

For double lymphatic vessel endothelial hyaluronan receptor-1 (LYVE-1) and CD31 immunostainings, corneas were incubated overnight with polyclonal goat antimouse LYVE-1 (1/200; R\&D Systems, Abingdon, UK) and rat antimouse CD31 (1/200; BD Biosciences Pharmingen, San Jose, CA). Then incubations with Alexa Fluor 488-coupled rabbit antigoat antibody (1/200; Molecular Probes, Merelbeke, Belgium) and Alexa Fluor 546-coupled goat antirat (1/200; Molecular Probes) were performed for 2 hours. For double LYVE-1$\mathrm{CD} 11 \mathrm{~b}$ or $\mathrm{F} 4 / 80$ immunostainings, corneas were incubated overnight with polyclonal goat antimouse LYVE-1 and biotincoupled rat antimouse CD11b (1/250; BD Biosciences) or rat antimouse F4/80 (1/100; Abcam, Cambridge, UK). Alexa Fluor 488-coupled rabbit antigoat antibody (1/200; Molecular Probes) and biotin-coupled rabbit antirat immunoglobulin (1/ 400; Dako, Glostrup, Denmark) were added for 2 hours, followed by 2 hours incubation with Alexa Fluor 555-coupled streptavidin (1/500; Molecular Probes).

Corneas flat-mounted on a microscope slide with Vectashield mounting medium (Vector Laboratories, Burlingame, CA) were examined with a fluorescent microscope (AH3RFCA; Olympus, Hamburg, Germany). Pictures were taken at $\times 40$ magnification and assembled to reconstitute the whole cornea picture with Microsoft Image Composite Editor (Microsoft Corporation, Santa Rosa, CA).

\section{Computerized Methods of Quantification}

The previously described computerized quantification method of lymphatic network developing in the cornea was extended to blood vessels. ${ }^{21}$ Image processing algorithms were first developed in order to extract blood and lymphatic vessels from the background. This resulted in two independent binary images, in which lymphatic and blood vessels were represented by white pixels (pixels value equal to 1 ) on a black background (pixels value equal to 0 ). After binarization, the following automatic measurements were performed: (1) area density defined as the surface covered by vessels reported to total cornea area; (2) end point density defined as the number of vessel extremities per corneal area unit (this parameter indicates the increase/decrease of sprout tips); (3) branching density, defined as the number of vessel branchings per corneal area unit, which indicates the structure degree of complexity; (4) length density, which represents the cumulative length of vessels; (5) maximal length of vessels, which indicates the largest migration distance; and (6) spatial distribution of vessels in relation to the limbal vessel. This distribution gives local information about the number of vessels at each given position in relation to the limbal vessel.

For inflammatory cell quantification (red labeling), the contrast was first enhanced on original red(R)-green(G)-blue(B) color image by combining color components as 2R-G-B images. Heterogeneous illumination was further corrected using a morphological tool (top-hat transformation). ${ }^{24}$ Finally, an automatic threshold maximizing the global average contrast across the picture ${ }^{25}$ was applied to obtain a binary image with intensity 1 and 0 , respectively for inflammatory cells and background. A 0.8-mm thick ring, comprised between the edge of the limbus and the center of the cornea, was arbitrary defined to eliminate unspecific staining present in the center of the cornea and resulting from cauterization. Cell density was calculated as the ratio of pixels belonging to inflammatory cells and the total ring area.

Image processing and measurements were performed using Aphelion 3.2 software (Adcis, Saint-Contest, France) and image analysis toolbox of Matlab 7.9 software (The Mathworks, Inc., Natick, MA).

\section{Macrophage Culture}

Peritoneal lavage was performed to isolate primary macrophages from $\mathrm{C} 57 \mathrm{BL} / 6$ mice intraperitoneally injected with $4 \%$ thioglycollate (T-9032; Sigma-Aldrich, St. Louis, MO) 5 days 
TABLE. Sequences of Primers Used for RT-PCR Experiments

\begin{tabular}{|c|c|c|c|c|}
\hline Gene & Primer Sequence & Hybrid. Temperature & Cycles & Length \\
\hline VEGF-A & $\begin{array}{l}\text { Sense: 5'-CCTGGTGGACATCTTCCAGGAGTA-3' } \\
\text { Anti-sense: 5'-CTCACCGCCTCCTGTCACA-3' }\end{array}$ & $66^{\circ} \mathrm{C}$ & 25 & $275 \mathrm{bp}$ \\
\hline VEGF-C & $\begin{array}{l}\text { Sense: 5'-GTAAAAACAAACTTTTCCCTAATTC-3' } \\
\text { Anti-sense: 5'-TTTAAGGAAGCACTTCTGTGTGT-3' }\end{array}$ & $60^{\circ} \mathrm{C}$ & 30 & 161 bp \\
\hline VEGF-D & $\begin{array}{l}\text { Sense: 5'-GGTTGTCTTCCCCAGCTC-3' } \\
\text { Anti-sense: 5'-TCTCGCTCAGCATCCCATC-3' }\end{array}$ & $55^{\circ} \mathrm{C}$ & 30 & 129 bp \\
\hline PIGF & $\begin{array}{l}\text { Sense: 5'-AGATCTTGAAGATTCCCCCCA-3' } \\
\text { Anti-sense: 5'-TTCCCCTTGGTTTTCCTCCTT-3' }\end{array}$ & $58^{\circ} \mathrm{C}$ & 33 & $130 \mathrm{bp}$ \\
\hline sVEGFR-1 & $\begin{array}{l}\text { Sense: 5'-AGGTGAGCACTGCGGCA-3' } \\
\text { Anti-sense: 5'-ATGAGTCCTTTAATGTTTGAC-3' }\end{array}$ & $60^{\circ} \mathrm{C}$ & 28 & $106 \mathrm{bp}$ \\
\hline sVEGFR-2 & $\begin{array}{l}\text { Sense: 5'-CACCAGTTTGCAAGAACTTGGATGCT-3' } \\
\text { Anti-sense: 5'-AATTCTGTCACCCAGGGATGC-3' }\end{array}$ & $62^{\circ} \mathrm{C}$ & 26 & $218 \mathrm{bp}$ \\
\hline GAPDH & $\begin{array}{l}\text { Sense: 5'-GCTGCCATTTGCAGTGGCAAAGT-3' } \\
\text { Anti-sense: 5'-GTGAAGACACCAGTAGACTCCAC-3' }\end{array}$ & $66^{\circ} \mathrm{C}$ & 21 & $247 \mathrm{bp}$ \\
\hline
\end{tabular}

earlier. Cells were cultured in serum-free RPMI-1640 (Lonza, Braine-l'Alleud, Belgium) for 1 hour at $37^{\circ} \mathrm{C}$. Non adherent cells were removed by 5 PBS washes. ${ }^{26}$ Adherent cells were cultured for 24 hours in serum-free RPMI-1640 before collecting conditioned medium. More than $80 \%$ macrophages were identified on cytospins.

\section{Aortic (ARA) and Lymphatic Ring Assays (LRA)}

Three-dimensional (3D) aortic or lymphatic ring cultures were performed as described previously. ${ }^{27-29}$ Briefly, aorta dissected from Wistar rats or thoracic duct dissected from C57BL/6 mice were cut into small fragments. The explants were embedded in interstitial type I collagen gel $(1.5 \mathrm{mg} / \mathrm{mL}$; Serva, Heidelberg, Germany) and cultured in MCDB131 (Invitrogen, Merelbeke, Belgium). For lymphatic ring assay, medium was supplemented with 2\% Ultroser G (BioSepra, Cergy Saint Christophe, France) and explants were cultured under hypoxic conditions $\left(5 \% \mathrm{O}_{2}\right.$, $5 \% \mathrm{CO}_{2}$, and $90 \% \mathrm{~N}_{2}$ ). In some conditions, $20 \mathrm{nM}$ sunitinib (LC Laboratories, Woburn, MA) or $10 \%$ macrophage conditioned medium was added to culture medium. VEGF-A $(20 \mathrm{ng} / \mathrm{mL}$; Peprotech, London, UK) was used as positive control in the aortic ring assays. Pictures were taken at the indicated times (6-13 days) and computerized quantifications were performed on binary images as described previously. ${ }^{29,30}$ Briefly, a grid comprised of concentric rings was generated by successive increments at fixed intervals of explant boundary. Then, the number of microvessel grid intersections was counted and plotted versus the distance from the ring to determine microvessel distribution. At least five images per experimental condition were used.

\section{Reverse Transcription-Polymerase Chain Reaction}

Frozen corneas were pulverized (MagNA Lyser; Roche, Indianapolis, IN) and total RNA was extracted with a kit (RNeasy; Qiagen, Hilden, Germany) according to the manufacturer's protocol. Cultured cells were washed with cold PBS and total RNA was extracted with a kit (Roche, Indianapolis, IN) according to the manufacturer's protocol. VEGF-A, VEGF-C, VEGF-D, placental growth factor (PIGF), sVEGFR-1, sVEGFR-2, and glyceraldehyde-3-phosphate deshydrogenase (GAPDH) mRNA were amplified with a 10-ng aliquot of total RNA using an amplification kit (GeneAmp Thermostable rTth Reverse Transcriptase RNA PCR Kit; Roche, Branchburg, Germany). Reverse transcription was performed at $70^{\circ} \mathrm{C}$ for 15 minutes followed by RNA-DNA heteroduplexe denaturation at $94^{\circ} \mathrm{C}$. Amplification started by a 20 second cycle at $94^{\circ} \mathrm{C}, 20$ seconds at hybridation temperature, and finally 20 seconds at $72^{\circ} \mathrm{C}$. RTPCR products were resolved in $10 \%$ acrylamide gels after staining with Gel Star (Cambrex, East Rutherford, NJ). The primer sequences, hybridation temperatures, number of cycles, and the length of the RT-PCR products are described in the Table.

\section{Endothelial Cell Culture and Western Blotting}

LECs purchased from Lonza (HMVEC-dLy; Braine-l'Alleud, Belgium) were cultured in endothelial growth medium microvascular (EGM2-MV) medium (Lonza) supplemented with 5\% fetal calf serum (FCS) and L-glutamine (Invitrogen) until confluence was reached. Cells were then rinsed with PBS and placed for 24 hours in endothelial basal medium (EBM2) serum-free medium (Lonza). Medium was then replaced by EBM2 containing $20 \mathrm{nM}$ sunitinib or $0.1 \%$ DMSO for 2 hours, and cells were stimulated with $10 \%$ macrophage conditioned medium for 10 minutes. In some samples, macrophage conditioned medium was preincubated for 1 hour with 500 ng/mL sVEGFR-1 or sVEGFR-3 (R\&D Systems) to trap VEGF-A or VEGF-C and -D, respectively. Cells were rinsed with ice cold PBS and lysed with radioimmunoprecipitation assay (RIPA) buffer containing phosphatase and protease inhibitors (Roche, Vilvoorde, Belgium). Samples were dissolved in SDS buffer and migrated on $12 \%$ SDS-PAGE gel before being transferred onto a polyvinyl difluoride (PVDF) membrane. After 1 hour blocking in $1 \%$ casein, phosphorylated, and total proteins were detected by $4^{\circ} \mathrm{C}$ overnight incubation with the respective antibodies, followed by 1 hour room temperature incubation in HRPcoupled secondary antibody (Cell Signaling, San Diego, CA) and ECL revelation in LAS4000 imager (Fujifilm, Tokyo, Japan). The following antibodies were used: rabbit monoclonal antiphospho-Akt, Akt, phospho-ERK1/2, ERK1/2, phosphoVEGFR-2 and VEGFR-2 (Cell Signaling).

\section{Proliferation and Tubulogenesis Assay}

For proliferation assay, $4 \times 10^{3}$ LECs (HMVEC-dLy; Lonza) were seeded in wells of 96-well plate and were grown overnight in EGM2-MV medium (Lonza). Medium was then replaced by serum-free EBM2 medium (Lonza) for 2 hours and cells were stimulated with $50 \%$ macrophage conditioned medium for 48 hours containing $20 \mathrm{nM}$ sunitinib or not. In some samples, macrophage conditioned medium was preincubated for 1 hour with $1 \mu \mathrm{g} / \mathrm{mL}$ sVEGFR-1 or sVEGFR-3 (R\&D Systems) to trap VEGF-A or VEGF-C and -D, respectively. Cell proliferation was 
then assessed using WST-1 proliferation kit (Roche, Indianapolis, IN) according to the manufacturer's protocol.

For tubulogenesis assay, $3 \times 10^{5}$ LECs were seeded on a type I collagen layer $(1 \mathrm{mg} / \mathrm{ml}$, Collagen R; Serva Electrophoresis, Heidelberg, Germany) in a 12-well plate. Cells were grown for 24 hours in EGM2-MV medium. Medium was then removed and a second layer of collagen was added over the cells. Finally, collagen-embedded LECs were incubated in $500 \mu \mathrm{L}$ of serumfree EBM2 medium containing or not 50\% macrophage conditioned medium and/or $20 \mathrm{nM}$ sunitinib. After 6 hours, five pictures per well were captured with a phase-contrast microscope (Axiovert 25; Carl Zeiss Microscopy, Zaventem, Belgium) coupled to an Axiocam color digital camera (Carl Zeiss) and tube length was measured.

\section{RESULTS}

\section{Time Course Corneal Lymph/Angiogenesis}

Corneal NV was induced in $\mathrm{C} 57 \mathrm{BL} / 6$ mice by thermal cauterization. Mice were time course sacrificed to concomitantly evaluate the progression of blood and lymphatic networks developing in the cornea by double LYVE-1-CD31 immunostaining on whole-mounts (Fig. 1A). In healthy corneas (Day 0), blood and lymphatic vessels were restricted to the limbus but some lymphatics extended in the cornea as previously described. ${ }^{31}$ Three days after cauterization, significant hemangiogenesis was observed that persisted until day 21. This was followed by a strong blood vessel regression observed after 40 days. The lymphangiogenic process took place more slowly with some short sproutings appearing on lymphatic vessels at day 3 (Fig. 1A). Lymphatic vessels strongly expanded between days 7 and 21. Lymphatic vessel regression occurred in a lesser extent than the blood vessel ones. A significant number of lymphatic vessels were still observed in the center of corneas after 40 days (Fig. 1A). These vascular changes were quantified through an original computerized method (Fig. 1B), and objectivized through four parameters: area density, length density, branching density, and end point (sprout tip) density. Regarding hemangiogenesis, all these parameters evidenced a linear and rapid blood vessel growth that occurred until day 21 and was followed by a strong regression leading to a bell shape curve. A latency phase of 5 to 7 days was observed for lymphangiogenesis, followed by a rapid growth of lymphatic vessels, and finally, a regression phase (Figs. 1C-F). The number of lymphatic vessels being far inferior to blood vessel ones, lymphatic area density and length density were much lower than blood vessel densities. Beside area and length densities that provide an overall information on blood and lymphatic vessel growth (Figs. 1C, 1D), branching and end point densities give quantitative information about the complexity of these vascular networks (Figs. 1E, 1F). This quantification underlines that blood vessels presented much more branchings and end points than lymphatics reflecting a much more complex and ramified plexus.

\section{Sunitinib Inhibits Both Corneal Hemangiogenesis and Lymphangiogenesis}

Corneal NV was induced in mice administered with $40 \mathrm{mg} / \mathrm{kg}$ sunitinib by daily oral gavage. Mice were sacrificed 6, 11, or 17 days after cauterization and corneas were immunostained for LYVE-1 and CD31. At early time points (days 6 and 11), sunitinib did not inhibit the sprouting of blood vessels and similar number of end points and branchings were observed in the two experimental groups (Figs. 2A-D). Nevertheless, although similar blood vessel density was observed near the limbus, the maximal distance of cell migration (Lmax) was significantly reduced at day 11 upon sunitinib treatment (Lmax $=0.82 \pm 0.05$ in controls versus $0.62 \pm 0.04$ in sunitinib treated $P=0.0012$ ) (Fig. 2E). This indicates that sunitinib inhibited blood vessel elongation at early time points. At day 17 , all parameters measured were 1.8 -fold decreased in sunitinib-treated mice compared with controls $(P<0.001)$ (Figs. 2A-E). Notably, the density of vessels closed to the limbus (distance below $0.4 \mathrm{~mm}$ ) was drastically decreased suggesting a regression of neoformed blood vessels (Fig. 2F). Therefore, the computer-assisted quantification reveals that sunitinib did not influence the sprouting of new vessels from the limbal vessels, but mainly impaired vessel elongation and induced neoformed vessel regression. These observations are consistent with the capacity of sunitinib to block VEGF and PDGF receptors. Regarding lymphatic vasculature, all parameters measured were reduced from day 6 to day $17(P<0.001)$ (Figs. 2G-K), suggesting a rapid inhibition of both lymphatic vessel sprouting and elongation. This is supported, at least, by the decreased vessel density detected upon sunitinib treatment, at a distance lower than $0.4 \mathrm{~mm}$ from the limbus.

\section{Sunitinib Inhibits Inflammatory Cell Recruitment in the Cornea}

We then evaluated the recruitment of inflammatory cells after cornea cauterization in mice treated or not with sunitinib through immunostaining directed against $\mathrm{CD} 11 \mathrm{~b}$, which is a general marker of inflammatory cells, or F4/80, which is more specific of monocytic cells such as macrophages. Inflammatory cells, especially macrophages, are indeed known to play an important role in cornea NV, being at least a source of prolymph/angiogenic factors. ${ }^{12}$ Through a preliminar time course analysis of inflammatory cell recruitment in cauterized cornea, we previously observed an early CD11b + cell infiltration peaking at 1 and 2 days post cauterization. F4/80+ cells appeared later, from day 3 to day 14 post cauterization. Double LYVE-1-CD11b and LYVE-1-F4/80 whole-mount immunostainings were conducted at different time points (Fig. 3B). Immunostaining quantification was performed after image binarization (Fig. 3A). Strong CD11b+ cell infiltration was observed in both experimental groups and quantification did not revealed any difference between mice treated with sunitinib and controls, 2 days or 7 days after cauterization (Figs. 3B, 3C). An important recruitment of F4/80+ cells was observed in control corneas after 3, 7, and 14 days. Notably, a reduction of $\mathrm{F} 4 / 80+$ cell infiltration was already observed at day 3 and persisted until day 14 in corneas from sunitinibtreated mice $(P<0.001$ for day $3, P<0.05$ for day $7, P<0.01$ for day 14) (Fig. 3C).

Since inflammatory cells are major producers of prolymph/ angiogenic molecules, notably VEGF-A, -C, and -D, and PIGF, we analyzed the expression levels of these factors by RT-PCR analysis of mRNA extracted from corneas treated or not with sunitinib, as well as the expression level of sVEGFR-1 and sVEGFR-2 reported as a regulator of angiogenesis and lymphangiogenesis, respectively. ${ }^{2,3}$ At day 7, VEGF-A mRNA levels were reduced 2-fold in sunitinib treated corneas after cauterization $(P<0.01)$ (Supplementary Data S1A, S1B). VEGFC expression was reduced by $35 \%$ at day 21 , but not modulated at earlier time point $(P<0.01)$ (Supplementary Data S1C, S1D). VEGF-D and PIGF mRNA levels were weak and no difference was observed between the experimental groups (Supplementary Data S1A, S1B). Identically, sVEGFR-1 and sVEGFR-2 expressions were not affected by sunitinib treatment (Supplementary Data S1A, S1B). 

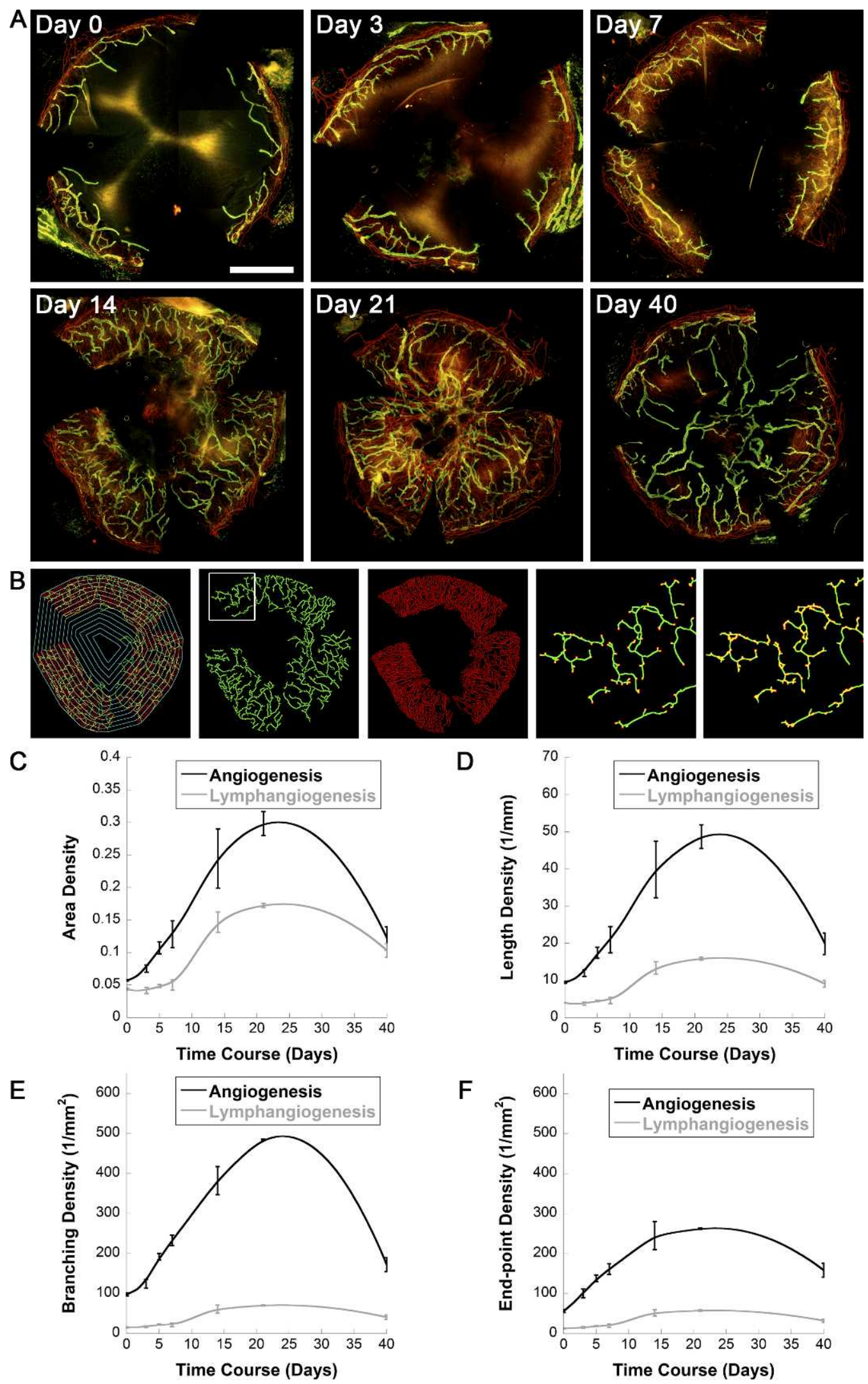

Figure 1. Time course corneal lymph/angiogenesis development after thermal cauterization. (A) Blood (red) and lymphatic (green) vessels were evidenced by whole-mount immunostaining directed against CD31 and LYVE-1, respectively, in healthy corneas (Day 0), or 3, 7, 14, 21, or 40 days after cornea cauterization. (B) Computer-assisted quantification was based on the splitting of red and green channels to dissociate blood from lymphatic vessels. A grid (white) was applied on each cornea picture to establish the distribution curves of capillaries around the limbal vessels (left panel). The number of end points and branchings was determined after image binarization for blood and lymphatic vessels. The two panels on the right illustrate, at higher magnification, end points and branchings automatically delineated by red/yellow spots. (C-F) Parameters characterizing 
the blood (black curve) and lymphatic (grey curve) vasculatures were normalized to the total area of the cornea (density) and include: area covered by neoformed vessels (area density, [C]); cumulative length of the vessels (length density, $[\mathbf{D}]$ ); number of bifurcations (branching density, $[\mathbf{E}]$ ); number of sprout tips (end point density, [F]). Bar represents $1 \mathrm{~mm}$

\section{Sunitinib Inhibits In Vitro Hemangiogenesis but Not Lymphangiogenesis}

We next performed in vitro aortic and lymphatic ring assays to evaluate whether sunitinib could directly inhibit angiogenesis and lymphangiogenesis (Fig. 4). By adding increasing concentrations of sunitinib (from 1-1000 $\mathrm{nM}$ ) in the culture medium of the aortic rings, we evidenced a strong inhibitory effect on angiogenesis from $10 \mathrm{nM}$ doses (Fig. 4D). Upon VEGF-A stimulation of the aortic ring, the angiogenic response was enhanced (Figs. 4A [left and middle panels], 4B, 4D). This VEGF-A-mediated angiogenic activity was drastically reduced by the addition of $20 \mathrm{nM}$ sunitinib (Figs. 4A, 4B, 4D). In other experiments, aortic rings were first cultured with sunitinib during 6 days leading to a strong inhibition of endothelial cell spreading out. When the culture medium was replaced by sunitinib-free medium, blood capillary growth was rescued demonstrating that the antiangiogenic effects of sunitinib does not rely on a toxic effect on endothelial cells (data not shown). Surprisingly, in the lymphatic ring assay, sunitinib failed to affect lymphangiogenesis, at concentrations ranging from 1 to $1000 \mathrm{nM}$ (Figs. 4A [right panels], 4C, 4E). These data suggest that sunitinib does not exert a direct inhibitory effect on LECs. It is worth mentioning that the impact of sunitinib in the lymphatic ring assay has been tested in the presence of $2 \%$ Ultroser leading to an optimal lymphangiogenic reaction. Therefore, the failure of sunitinib to block LEC sprouting cannot be ascribed to too low proliferation rate.

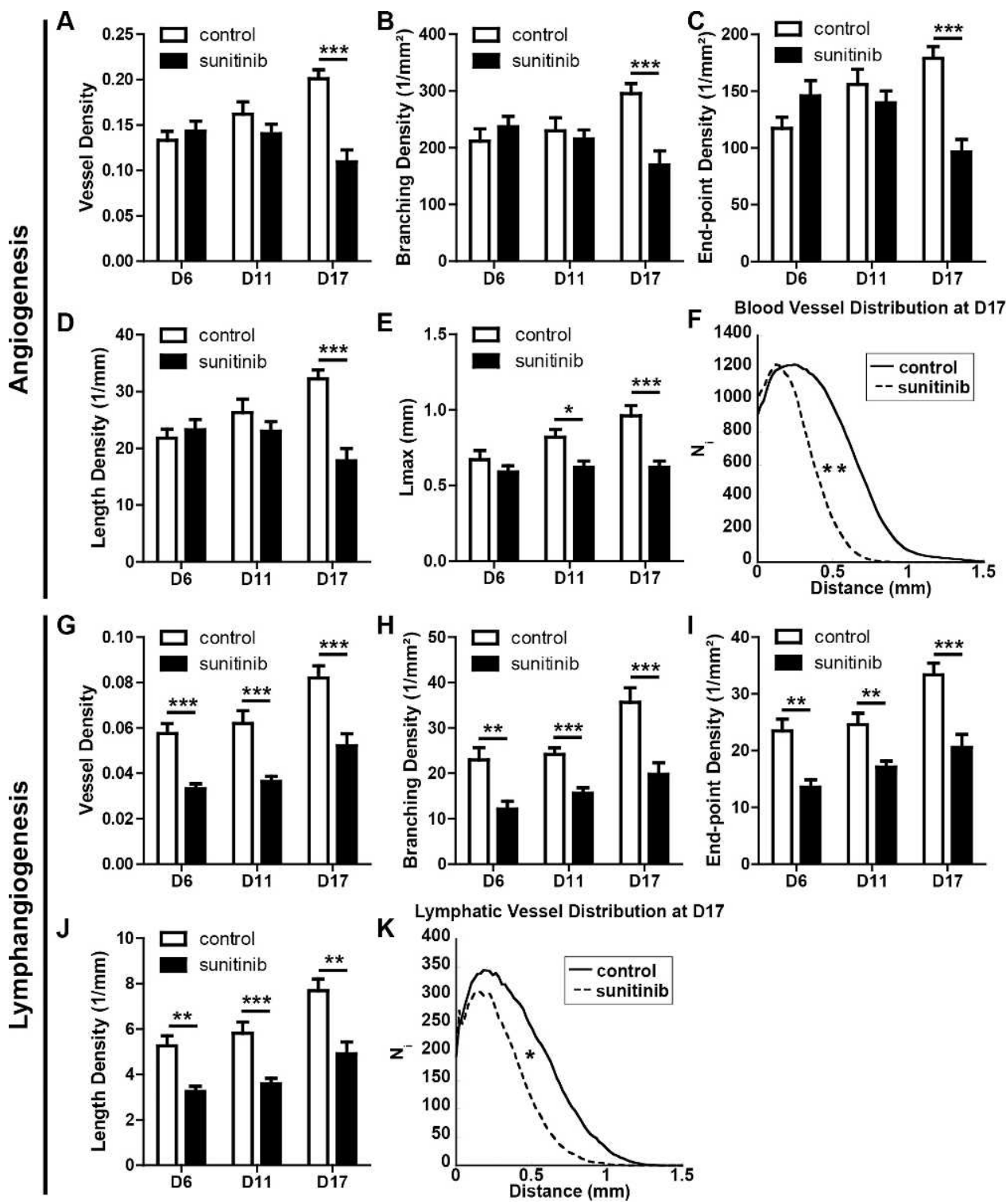

Figure 2. Sunitinib administration inhibits corneal neovascularization. Computer-assisted quantification of blood (A-F) and lymphatic (G-K) vascular networks developing in the cornea after cauterization, in mice treated with sunitinib (black columns) or not (white columns). Vessel area $(\mathbf{A}, \mathbf{G})$, branching $(\mathbf{B}, \mathbf{H})$, end point $(\mathbf{C}, \mathbf{I})$, and total length $(\mathbf{D}, \mathbf{J})$ densities, as well as maximal length of vessels (Lmax) (E), and vessel distributions (F, K) were evaluated. ${ }^{*} P<0.05 ;{ }^{* *} P<0.01 ;{ }^{* * *} P<0.001$. 
A
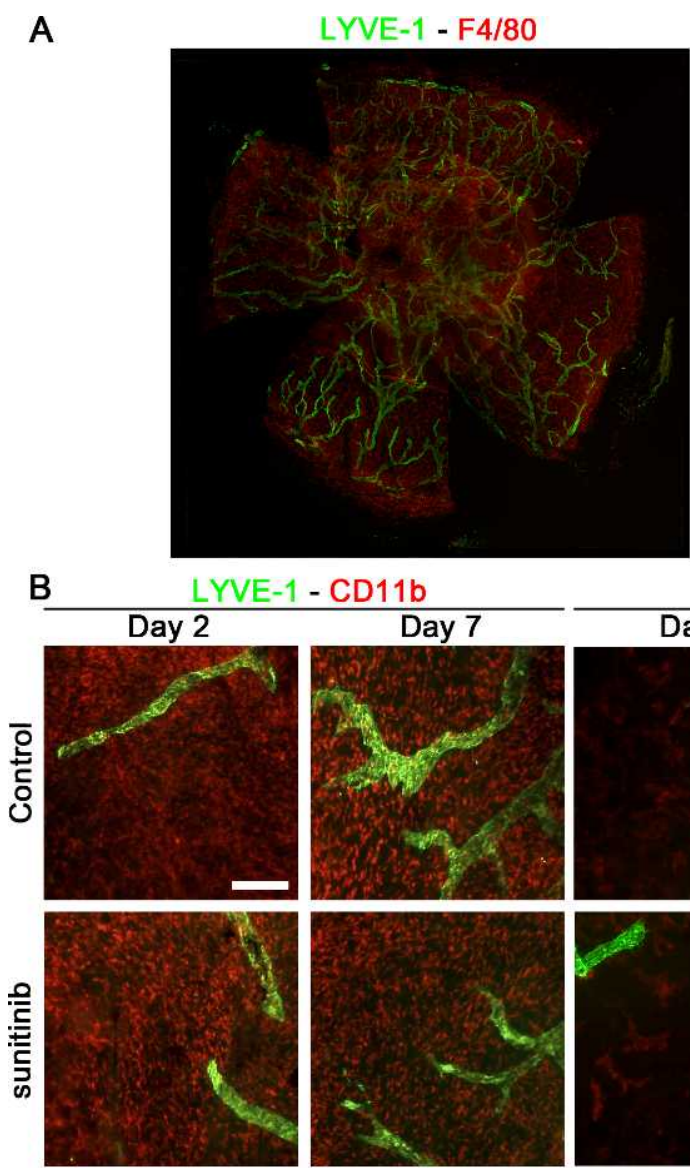

C
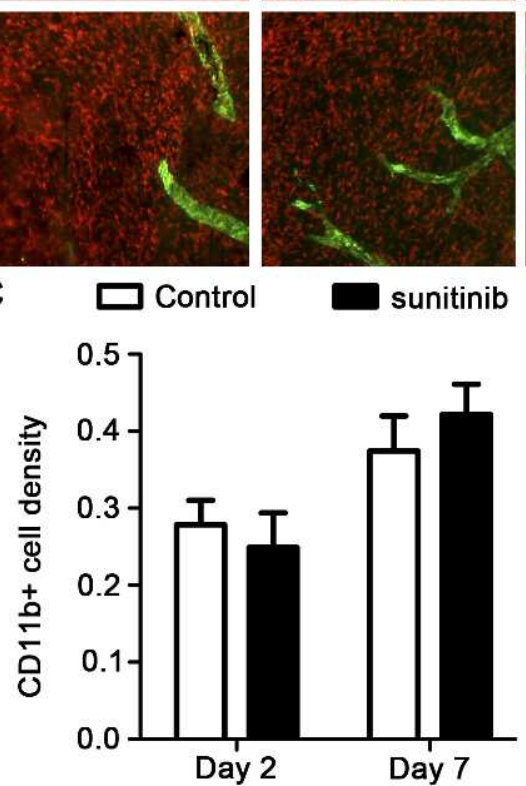

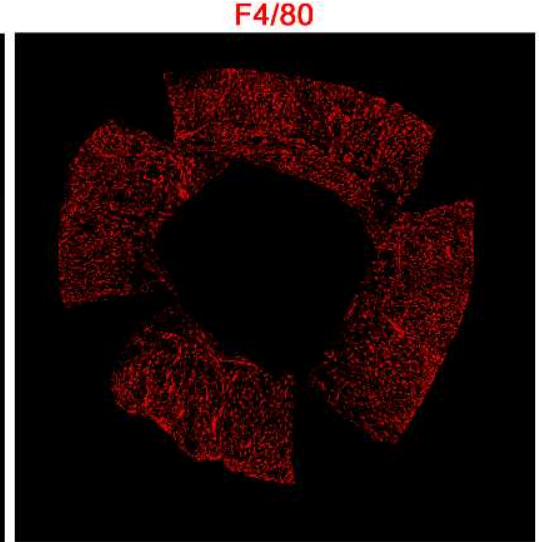

LYVE-1 - F4/80

Day 7
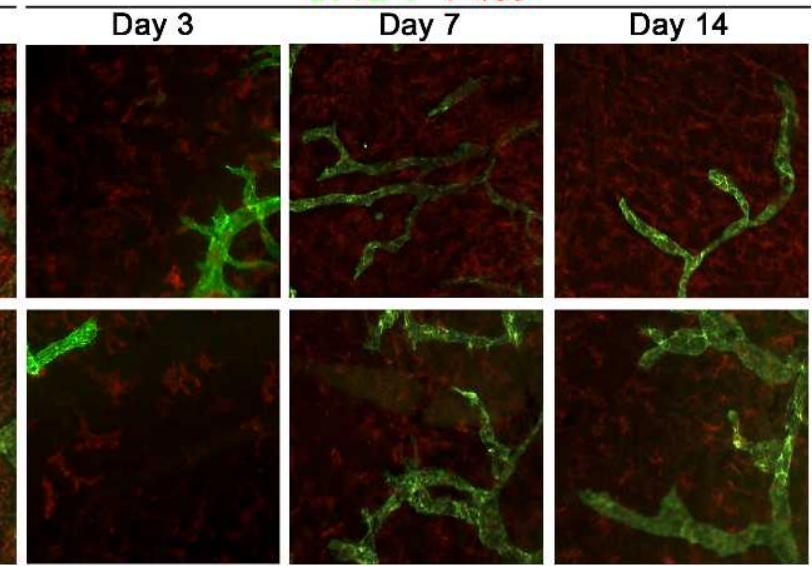

$\square$ Control
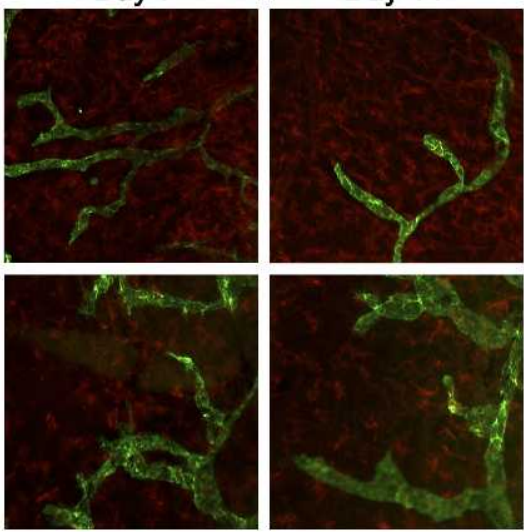

sunitinib

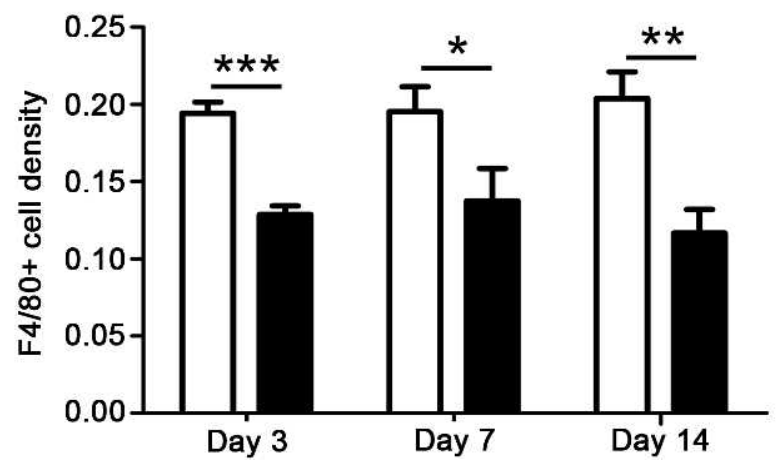

FIgURE 3. Sunitinib affects F4/80+ cell infiltration in inflamed corneas. (A) Computer-assisted F4/80 immunostaining (red) quantification on double whole-mount immunostaining is performed after binarization by reporting the surface covered by $\mathrm{F} 4 / 80+$ cells to the total surface of the cornea. A distance of $800 \mu \mathrm{m}$ from the outer limit of the cornea was considered for quantification (right panel). (B) Double LYVE-1 - CD11b (left panel) or F4/ 80 (right panels) whole-mount immunostainings were performed at indicated time points, on corneas of mice treated with sunitinib (lower panels) or not (upper panels). (C) Histograms representing quantification of CD11b+ (left panel) and $\mathrm{F} 4 / 80+($ right panel) inflammatory cell infiltration in inflamed corneas. Bar represents $100 \mu \mathrm{m} .{ }^{*} P<0.05 ;{ }^{* * *} P<0.01$.

\section{Sunitinib Inhibits Macrophage-Induced Lymph/ Angiogenesis}

To next evaluate the role of macrophages on lymph/ angiogenesis in vitro, macrophages isolated from the peritoneal cavity were treated overnight with $20 \mathrm{nM}$ sunitinib or $0.1 \%$ DMSO. RT-PCR analyses revealed high expression levels of VEGF-A, VEGF-C, and VEGF-D without any modulation by sunitinib treatment (data not shown). Media conditioned by macrophages were then used in in vitro experiments of aortic and lymphatic ring assays (Fig. 5). The growth of aortic explants cultured in the presence of macrophage conditioned medium was increased as compared with controls and nearly similar to that of explants exposed to $10 \mathrm{ng} / \mathrm{mL}$ recombinant VEGF-A (Figs. 5A, 5B). Ring treatment with $20 \mathrm{nM}$ sunitinib induced a significant reduction of capillary outgrowth compared with control rings (Figs. 5A, 5B).

In an identical manner, macrophage conditioned medium strongly stimulated lymphatic ring growth (Figs. 5C, 5D). While sunitinib did not reduce the growth of nonstimulated lymphatic rings (Figs. 4A, 4C, 4E), a strong inhibition of macrophage-induced lymphangiogenesis was observed (Figs. 
A

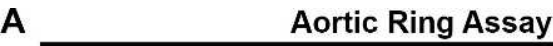

Lymphatic
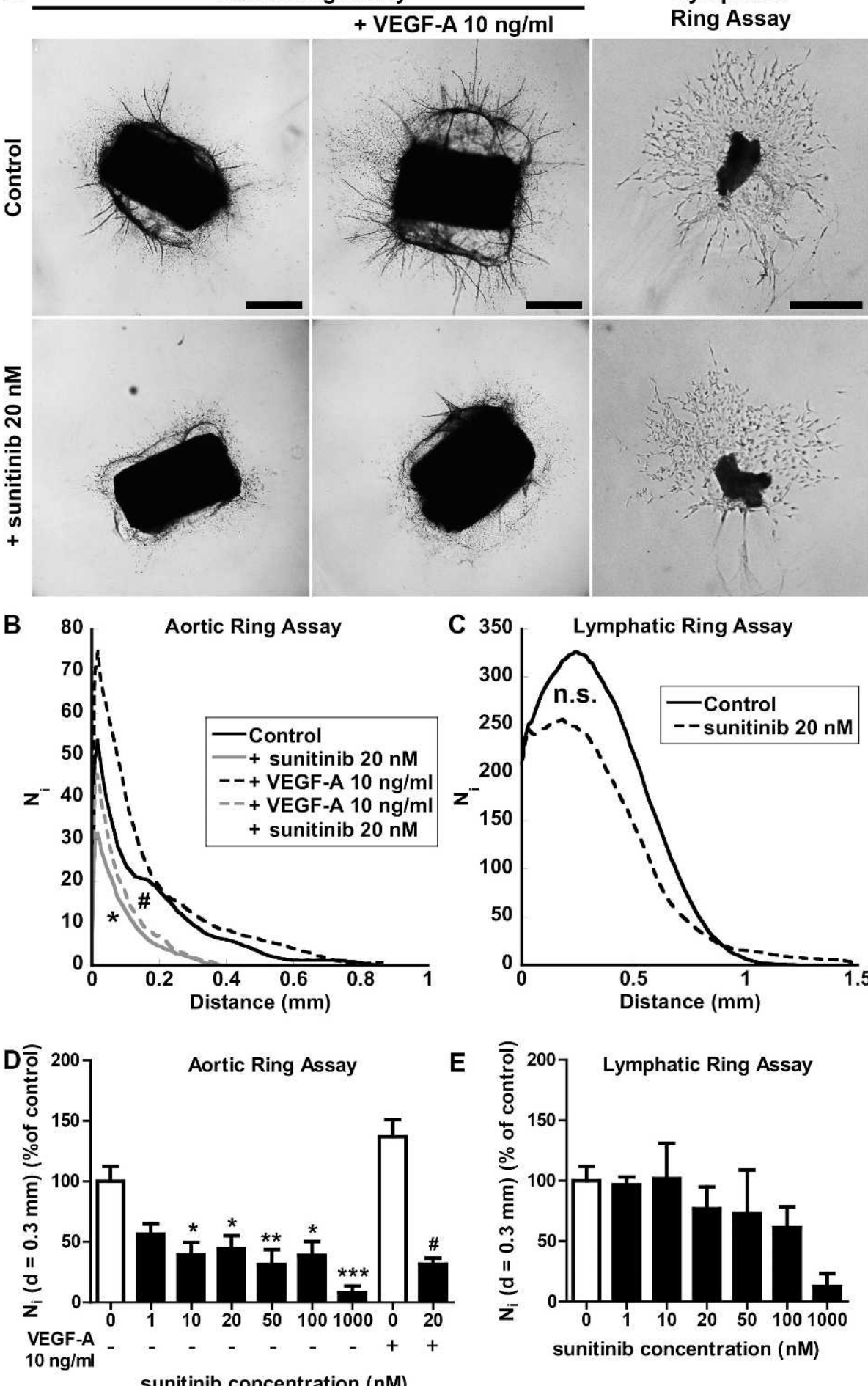

sunitinib concentration (nM)

FiguRE 4. Sunitinib inhibits in vitro angiogenesis, but not lymphangiogenesis. (A) Mouse aorta or lymphatic duct explants embedded in type I collagen gel were cultured for 9 days in the absence (control) or presence of $20 \mathrm{nM}$ sunitinib ( + sunitinib). (B, C) For quantification, grid corresponding to successive increments at fixed intervals of explant boundary was used on binarized images and the number of microvessel grid intersections $\left(\mathrm{N}_{\mathrm{i}}\right.$ ) was quantified. (D, E) Aortic and lymphatic rings were cultured with increasing doses of sunitinib (ranging from $0-1000 \mathrm{nM}$ ). Histograms represent the number of vessels (Ni) present at a distance of $0.3 \mathrm{~mm}(\mathrm{~d}=0.3 \mathrm{~mm}) . \#,{ }^{*} \boldsymbol{P}<0.05 ;{ }^{* * *} \boldsymbol{P}<0.01 ;{ }^{* * * *} P<0.001\left({ }^{*}\right.$ compares sunitinib-treated conditions with control and \# compares VEGF-A $10 \mathrm{ng} / \mathrm{mL}+$ sunitinib condition with VEGF-A $10 \mathrm{ng} / \mathrm{mL}$ condition).

5C, 5D). Similarly, medium conditioned by macrophages promoted proliferation and tubulogenesis of isolated LECs (Supplementary Data S2). Sunitinib inhibited these stimulatory effects of macrophage conditioned medium (Supplementary
Data S2). To give mechanistic insights into sunitinib action on lymphangiogenesis, we preincubated macrophage conditioned medium with soluble forms of VEGFR-1 or VEGFR-3 to trap VEGF-A or VEGF-C, respectively. In these conditions, sVEGFR-1 

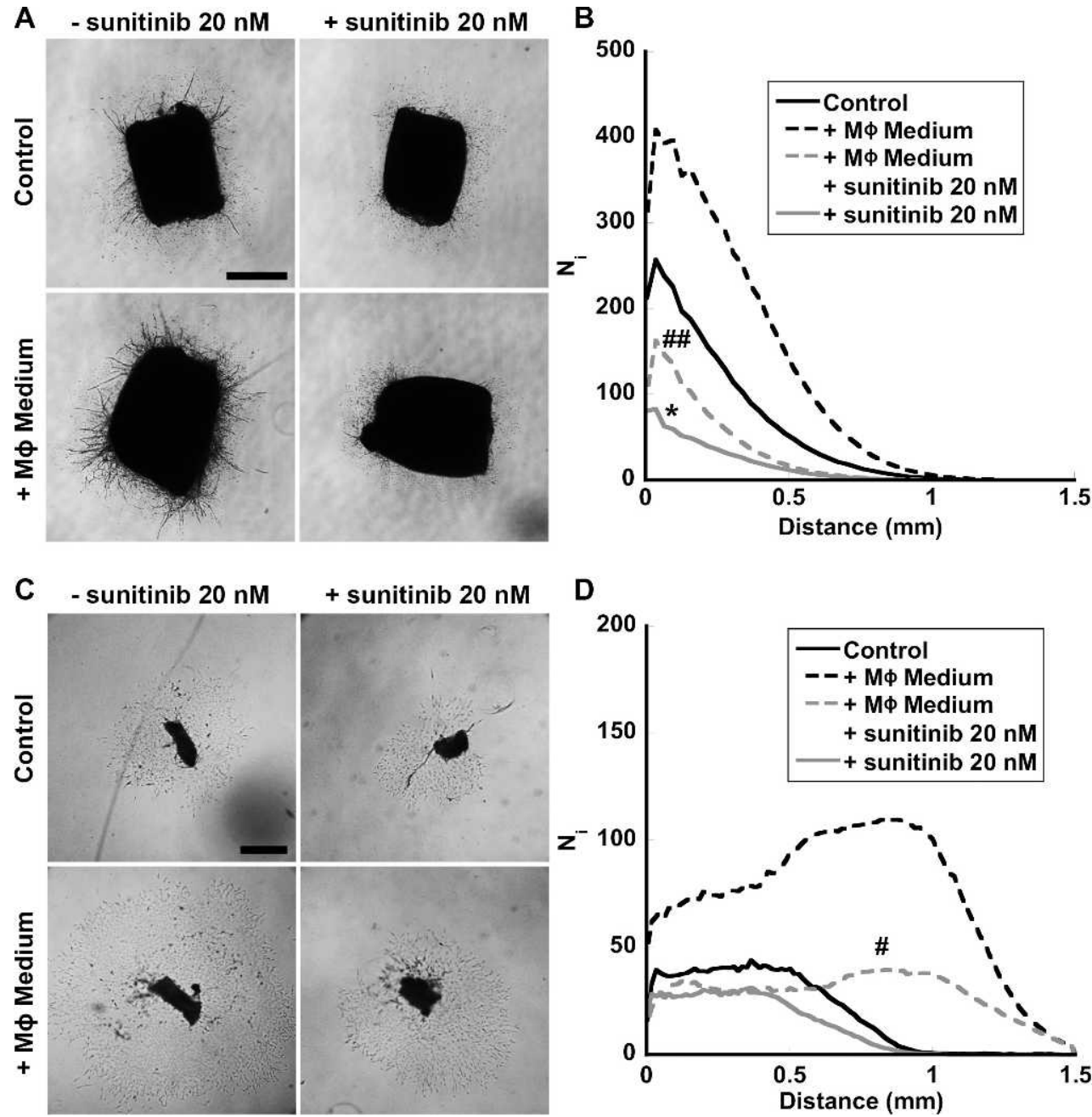

FiguRE 5. In vitro macrophage conditioned medium-enhanced lymph/angiogenesis are reduced by sunitinib. (A, C) Aortic (A) or lymphatic (C) rings were cultured in absence (control) or presence of $10 \%$ macrophage conditioned medium (M $\phi$ medium) with ( + sunitinib) or without $(-$ sunitinib) sunitinib (20 nM). (B, D) For quantification, grid corresponding to successive increments at fixed intervals of explant boundary was used on binarized images and the number of microvessel grid intersections $\left(\mathrm{N}_{\mathrm{i}}\right)$ was quantified on binarized images. $\#,{ }^{*} P<0.05$; \#\#P<0.01 (* compares sunitinib-treated condition with control and \# compares macrophage conditioned medium + sunitinib condition with macrophage conditioned medium condition)

significantly reduced LEC proliferation while sVEGFR-3 failed to do it, suggesting that VEGF-A is the major prolymphangiogenic factor secreted in the macrophage conditioned medium (Fig. 6). Concordantly, medium conditioned by macrophages induced the phosphorylation of VEGFR-2 and two downstream mediators (Akt and ERK1/2) as assessed by Western blot (Supplementary Data S3). Upon sunitinib treatment, the phosphorylation levels were drastically reduced demonstrating the implication of VEGFR-2 pathway in the observed effects. Moreover, conditioned medium preincubated with sVEGFR-1 induced weaker VEGFR-2 phosphorylation compared with control macrophage conditioned medium. Conversely, preincubation with sVEGFR-3 did not affect the level of phosphorylated proteins (Supplementary Data S3).

\section{Discussion}

Our study sheds light on the inhibition of corneal NV through the use of a broad spectrum tyrosine kinase inhibitor. We, here, provide evidence for combined antihemangiogenic and antilymphangiogenic potential of sunitinib, of interest for ocular disorders. Sunitinib induces a biphasic response on corneal NV with a rapid and early inhibition of lymphangiogenesis, followed by a blockade of hemangiogenesis. The lymphangiogenesis inhibitory action relies at least on its capacity to reduce inflammatory cell recruitment and to interfere with their secreted growth factors.

Pérez-Santonja and colleagues previously showed that topical sunitinib and bevacizumab administrations were able to inhibit corneal angiogenesis in a rabbit suture-induced model. ${ }^{19}$ However, corneal NV not only relies on hemangiogenesis, but also on lymphangiogenesis, which has been described as playing a major role in cornea graft rejection by providing a route for antigens and antigen presenting cells to regional lymph nodes. ${ }^{8,32}$ Our results provide evidence for a strong lymphangiogenesis inhibitory potential of sunitinib. Notably, this lymphangio-inhibitory action of sunitinib is exerted earlier than its antihemangiogenic effects. Our results are in line with the inhibitory effect of sunitinib on 


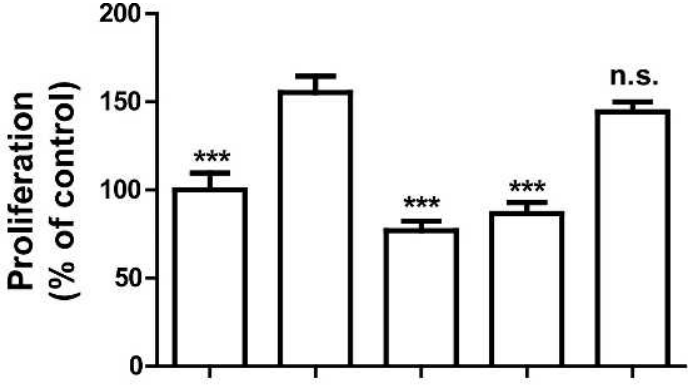

$\begin{array}{rrrrrr}\text { FBS } 0.5 \% & + & + & + & + & + \\ \text { MФ Medium } & - & + & + & + & + \\ \text { Sunitinib } 20 \mathrm{nM} & - & - & + & - & - \\ \text { SVEGFR-1 } 1 \mu \mathrm{M} & - & - & - & + & - \\ \text { sVEGFR-3 } 1 \mu \mathrm{M} & - & - & - & - & +\end{array}$

Figure 6. Soluble form of VEGFR-1 inhibits in vitro LEC proliferation induced by macrophage conditioned medium. LEC proliferation was assessed in the presence $(+)$ or absence $(-)$ of macrophage conditioned medium (M $\phi$ Medium), sunitinib, sVEGFR-1, or sVEGFR-3. ${ }^{* * *} P<$ 0.001 .

lymphangiogenesis observed in vivo in a murine breast cancer model. ${ }^{20}$ We also provide evidence that sunitinib, in addition to reducing blood and lymphatic vessel growth, is able to inhibit $\mathrm{F} 4 / 80+$ cell recruitment, but not $\mathrm{CD} 11 \mathrm{~b}+$ cell infiltration. Accordingly, other tyrosine kinase inhibitors, such as PTK787/ ZK222584 and ZK261991, have been reported to display similar inhibition of macrophage recruitment in corneal NV model. ${ }^{16}$ Reduced F4/80+ cell infiltration could result from the inhibition of VEGF-A/VEGFR-1 pathway. Infiltrating macrophages represent a major source of prolymph/angiogenic factors such as VEGF-A, -C, and -D, leading to an immune amplification. ${ }^{12}$ It has been reported that macrophages can also take part in the formation of lymphatic vessels by transdifferentiating into an endothelial phenotype and incorporating into the lymphatic wall. ${ }^{33,34}$ The reduced F4/80+ cell infiltration observed in our experimental model was associated to a reduction of corneal expression of VEGF-A and -C upon sunitinib treatment, although sunitinib treatment did not affect their expression in isolated macrophages. This observation suggests an indirect effect of sunitinib by interfering with F4/ $80+$ cell recruitment, thereby reducing the release of prolymphangiogenic factors. In addition to its impact on inflammatory cell recruitment, sunitinib is able to abrogate the effects of factors secreted by macrophages as demonstrated in the in vitro ring assays.

Surprisingly, although we observed a strong inhibition of lymphangiogenesis in vivo upon sunitinib treatment, no inhibitory effect was observed in vitro in the lymphatic ring assay. Notably, upon lymphatic ring stimulation with macrophage conditioned medium, sunitinib reduced LEC outgrowth to a level comparable of control conditions. This indicates that the effect of sunitinib in vivo relies on its capacity to interfere with prolymphangiogenic factors produced by macrophages. This concept is further supported by the capacity of sunitinib to block macrophage-mediated LEC proliferation and tube formation, as well as to inhibit the activation of VEGFR-2 pathway. The blockade of macrophage-mediated mitogenic effect by sVEGFR1 underlines the key contribution of VEGF-A in this process. In sharp contrast, hemangiogenesis was both inhibited in vivo and in vitro in the aortic ring assay, in basal conditions, as well as under VEGF-A or macrophage conditioned medium stimulation. It is worth mentioning that the aortic wall contains macrophages that could contribute to the endogenous release of angiogenic factors leading to an angiogenic response sensitive to sunitinib treatment. ${ }^{35,36}$

Treatments targeting lymph/angiogenesis have largely been developed in the past years and are now available to treat different tumor types and ocular pathologies affecting posterior segment of the eye. ${ }^{13}$ However, pathologic corneal NV still lacks specific management, while being major factor of vision impairment and strongly associated with the risk of cornea graft rejection. ${ }^{7,37}$ Angiogenesis and lymphangiogenesis are intricate processes in cornea $\mathrm{NV}$ and share common regulatory pathways.9 We provide data showing strong combined inhibition of corneal angiogenesis and lymphangiogenesis by sunitinib, suggesting a potential interest for corneal NV treatment. Importantly, a transient in vivo administration of sunitinib (during the first 6 days following cornea cauterization) was not sufficient to efficiently repress corneal NV. Similarly, no inhibitory effect of a transient sunitinib treatment was observed in vitro in the aortic ring assay. These data suggest a rapid regrowth of blood vessels after treatment withdrawal. This observation implies a sustained sunitinib exposure to treat ocular disorders. Previous studies demonstrated that corneal NV inhibition through VEGF-A blockade promoted cornea graft survival in murine model. ${ }^{15,38}$ Not only has VEGF-A been implicated in corneal NV, but also PDGF through its action on vessel stabilization, ${ }^{39}$ as well as VEGF-C and $-\mathrm{D}$ that activate lymphangiogenesis. ${ }^{40-42} \mathrm{~A}$ combined inhibition of VEGFR-2 and PDGFR in blocking corneal angiogenesis has proven more efficient than single VEGF-A pathway blockade. ${ }^{43,44}$ The use of multi-target tyrosine kinase inhibitors also provides higher survival rate for cornea engraftment. ${ }^{16}$ We, herein, provide evidence that the use of a multi-potent tyrosine kinase inhibitor such as sunitinib, both targeting effectors of hemangiogenesis and of lymphangiogenesis, could provide higher efficiency in the treatment of corneal NV.

In conclusion, our study provides evidence for strong antilymph/angiogenic potential of sunitinib in inflammationinduced corneal NV, acting through the inhibition of $\mathrm{F} 4 / 80+$ cell recruitment in inflamed corneas, thereby decreasing the secretion of VEGF-A and -C. The use of such a multi-target tyrosine kinase inhibitor could be considered for treatment in order to prevent cornea vascularization occurring in inflammatory conditions and to increase cornea graft survival in case of keratoplasty.

\section{Acknowledgments}

The authors thank the researchers from the Groupe Interdisciplinaire Génoprotéomique Appliquée (Animal Facility Platform, Imaging and Flow Cytometry Platform) and Guy Roland, Marie Dehuy, Nathalie Lefin, Isabelle Dasoul, Emilie Feyereisen, Patricia Gavitelli, and Laurette Volders.

Supported by grants from the Fonds de la Recherche Scientifique Médicale, the Fonds National de la Recherche Scientifique (FNRS, Belgium), the Fondation contre le Cancer, the Fonds spéciaux de la Recherche (University of Liège), the "Plan Cancer" (Belgium), the Centre Anticancéreux près l'Université de Liège, the Fonds Léon Fredericq (University of Liège), the Action de Recherche Concertée (ULg), the Interuniversity Attraction Poles ProgrammeBelgian Science Policy (Brussels, Belgium), and three Televie-FNRS grants (BD, CE, and LM).

Disclosure: B. Detry, None; S. Blacher, None; C. Erpicum, None; J. Paupert, None; L. Maertens, None; C. Maillard, None; C. Munaut, None; N.E. Sounni, None; V. Lambert, None; J.-M. Foidart, None; J.-M. Rakic, None; D. Cataldo, None; A. Noël, None 


\section{References}

1. Ellenberg D, Azar DT, Hallak JA, et al. Novel aspects of corneal angiogenic and lymphangiogenic privilege. Prog Retin Eye Res. 2010;29:208-248.

2. Albuquerque RJ, Hayashi T, Cho WG, et al. Alternatively spliced vascular endothelial growth factor receptor- 2 is an essential endogenous inhibitor of lymphatic vessel growth. Nat Med. 2009;15:1023-1030.

3. Ambati BK, Nozaki M, Singh N, et al. Corneal avascularity is due to soluble VEGF receptor-1. Nature. 2006;443:993-997.

4. Ambati BK, Patterson E, Jani P, et al. Soluble vascular endothelial growth factor receptor-1 contributes to the corneal antiangiogenic barrier. Br J Ophthalmol. 2007;91: 505-508.

5. Cursiefen C, Maruyama K, Bock F, et al. Thrombospondin 1 inhibits inflammatory lymphangiogenesis by CD36 ligation on monocytes. J Exp Med. 2011;208:1083-1092.

6. Azar DT. Corneal angiogenic privilege: angiogenic and antiangiogenic factors in corneal avascularity, vasculogenesis, and wound healing (an American Ophthalmological Society thesis). Trans Am Ophthalmol Soc. 2006;104:264-302.

7. Cursiefen C, Colin J, Dana R, et al. Consensus statement on indications for anti-angiogenic therapy in the management of corneal diseases associated with neovascularisation: outcome of an expert roundtable. Br J Ophthalmol. 2012;96:3-9.

8. Cursiefen $C$. Immune privilege and angiogenic privilege of the cornea. Chem Immunol Allergy. 2007;92:50-57.

9. Lohela M, Bry M, Tammela T, Alitalo K. VEGFs and receptors involved in angiogenesis versus lymphangiogenesis. Curr Opin Cell Biol. 2009;21:154-165.

10. Regenfuss B, Bock F, Parthasarathy A, Cursiefen C. Corneal (lymph)angiogenesis-from bedside to bench and back: a tribute to Judah Folkman. Lymphat Res Biol. 2008;6:191-201.

11. Wuest TR, Carr DJ. VEGF-A expression by HSV-1-infected cells drives corneal lymphangiogenesis. J Exp Med. 2010;207:101115

12. Cursiefen C, Chen L, Borges LP, et al. VEGF-A stimulates lymphangiogenesis and hemangiogenesis in inflammatory neovascularization via macrophage recruitment. J Clin Invest. 2004;113:1040-1050.

13. Noel A, Jost M, Lambert V, Lecomte J, Rakic JM. Antiangiogenic therapy of exudative age-related macular degeneration: current progress and emerging concepts. Trends $\mathrm{Mol}$ Med. 2007;13:345-352.

14. Bock F, Onderka J, Dietrich T, et al. Bevacizumab as a potent inhibitor of inflammatory corneal angiogenesis and lymphangiogenesis. Invest Ophthalmol Vis Sci. 2007;48:2545-2552.

15. Cursiefen C, Cao J, Chen L, et al. Inhibition of hemangiogenesis and lymphangiogenesis after normal-risk corneal transplantation by neutralizing VEGF promotes graft survival. Invest Ophthalmol Vis Sci. 2004;45:2666-2673.

16. Hos D, Bock F, Dietrich $\mathrm{T}$, et al. Inflammatory corneal (lymph)angiogenesis is blocked by VEGFR-tyrosine kinase inhibitor ZK 261991, resulting in improved graft survival after corneal transplantation. Invest Ophthalmol Vis Sci. 2008;49: 1836-1842.

17. Hos D, Saban DR, Bock F, et al. Suppression of inflammatory corneal lymphangiogenesis by application of topical corticosteroids. Arch Ophthalmol. 2011;129:445-452.

18. Faivre S, Demetri G, Sargent W, Raymond E. Molecular basis for sunitinib efficacy and future clinical development. Nat Rev Drug Discov. 2007;6:734-745.

19. Perez-Santonja JJ, Campos-Mollo E, Lledo-Riquelme M, Javaloy $\mathrm{J}$, Alio JL. Inhibition of corneal neovascularization by topical bevacizumab (anti-VEGF) and sunitinib (anti-VEGF and anti-
PDGF) in an animal model. Am J Ophthalmol. 2010;150:519528. e511.

20. Kodera Y, Katanasaka Y, Kitamura Y, et al. Sunitinib inhibits lymphatic endothelial cell functions and lymph node metastasis in a breast cancer model through inhibition of vascular endothelial growth factor receptor 3. Breast Cancer Res. 2011;13:R66.

21. Blacher S, Detry B, Bruyere F, Foidart JM, Noel A. Additional parameters for the morphometry of angiogenesis and lymphangiogenesis in corneal flat mounts. Exp Eye Res. 2009;89: 274-276.

22. Detry B, Bruyere F, Erpicum C, et al. Digging deeper into lymphatic vessel formation in vitro and in vivo. BMC Cell Biol. 2011;12:29.

23. Detry B, Erpicum C, Paupert J, et al. Matrix metalloproteinase2 governs lymphatic vessel formation as an interstitial collagenase. Blood. 2012;119:5048-5056.

24. Soille P. Morphological Image Analysis. Berlin: SpringerVerlag; 1999.

25. Kohler R. A segmentation system based on thresholding. Computer Graphics and Image Processing. 1981;319-338.

26. Davies JQ, Gordon S. Isolation and culture of human macrophages. Methods Mol Biol. 2005;290:105-116.

27. Berndt S, Blacher S, Perrier d'Hauterive S, et al. Chorionic gonadotropin stimulation of angiogenesis and pericyte recruitment. J Clin Endocrinol Metab. 2009;94:4567-4574.

28. Bruyere F, Melen-Lamalle L, Berndt S, Peulen O, Foidart JM, Noel A. The lymphatic ring assay: a 3D-culture model of lymphangiogenesis. Nat Protoc. 2008. doi:10.1038/nprot. 2008.86.

29. Bruyere F, Melen-Lamalle L, Blacher S, et al. Modeling lymphangiogenesis in a three-dimensional culture system. Nat Methods. 2008;5:431-437.

30. Blacher S, Devy L, Burbridge MF, et al. Improved quantification of angiogenesis in the rat aortic ring assay. Angiogenesis. 2001; 4:133-142.

31. Maruyama K, Nakazawa T, Cursiefen C, et al. The maintenance of lymphatic vessels in the cornea is dependent on the presence of macrophages. Invest Ophthalmol Vis Sci. 2012; 53:3145-3153.

32. Dana MR. Angiogenesis and lymphangiogenesis-implications for corneal immunity. Semin Ophthalmol. 2006;21:19-22.

33. Gordon EJ, Rao S, Pollard JW, Nutt SL, Lang RA, Harvey NL. Macrophages define dermal lymphatic vessel calibre during development by regulating lymphatic endothelial cell proliferation. Development. 2010;137:3899-3910.

34. Maruyama K, Ii M, Cursiefen C, et al. Inflammation-induced lymphangiogenesis in the cornea arises from CD11b-positive macrophages. J Clin Invest. 2005;115:2363-2372.

35. Gelati M, Aplin AC, Fogel E, Smith KD, Nicosia RF. The angiogenic response of the aorta to injury and inflammatory cytokines requires macrophages. J Immunol. 2008;181:57115719.

36. Nicosia RF, Zorzi P, Ligresti G, Morishita A, Aplin AC. Paracrine regulation of angiogenesis by different cell types in the aorta ring model. Int J Dev Biol. 2011;55:447-453.

37. Bachmann B, Taylor RS, Cursiefen C. Corneal neovascularization as a risk factor for graft failure and rejection after keratoplasty: an evidence-based meta-analysis. Ophthalmology. 2010;117:1300-1305. e1307.

38. Bachmann BO, Luetjen-Drecoll E, Bock F, et al. Transient postoperative vascular endothelial growth factor (VEGF)neutralisation improves graft survival in corneas with partly regressed inflammatory neovascularisation. Br J Ophthalmol. 2009;93:1075-1080. 
39. Dell S, Peters S, Muther P, Kociok N, Joussen AM. The role of PDGF receptor inhibitors and PI3-kinase signaling in the pathogenesis of corneal neovascularization. Invest Ophthalmol Vis Sci. 2006;47:1928-1937.

40. Alitalo K. The lymphatic vasculature in disease. Nat Med. 2011;17:1371-1380.

41. Cao R, Eriksson A, Kubo H, Alitalo K, Cao Y, Thyberg J. Comparative evaluation of FGF-2-, VEGF-A-, and VEGF-Cinduced angiogenesis, lymphangiogenesis, vascular fenestrations, and permeability. Circ Res. 2004;94:664-670.

42. Mimura T, Amano S, Usui T, Kaji Y, Oshika T, Ishii Y. Expression of vascular endothelial growth factor $\mathrm{C}$ and vascular endothe- lial growth factor receptor 3 in corneal lymphangiogenesis. Exp Eye Res. 2001;72:71-78.

43. Chaoran Z, Zhirong L, Gezhi X. Combination of vascular endothelial growth factor receptor/platelet-derived growth factor receptor inhibition markedly improves the antiangiogenic efficacy for advanced stage mouse corneal neovascularization. Graefes Arch Clin Exp Ophthalmol. 2011;249:14931501.

44. Jo N, Mailhos C, Ju M, et al. Inhibition of platelet-derived growth factor B signaling enhances the efficacy of anti-vascular endothelial growth factor therapy in multiple models of ocular neovascularization. Am J Patbol. 2006;168:2036-2053. 